\title{
Efectos de la restricción del pienso y suplementación con alfalfa sobre el rendimiento productivo, características de carcasa, índices cardiacos y parámetros hematológicos del pavo criado en condiciones hipobáricas
}

\author{
Effects of feed restriction and alfalfa supplementation on productive performance, \\ carcass characteristics, cardiac indices and haematological parameters of turkey \\ reared under hypobaric conditions
}

\author{
Manuel Paredes ${ }^{1,3}$, Corpus Tocas $^{1}$, Cristian Hobán $^{2}$, Pedro Ortiz ${ }^{2}$
}

\section{Resumen}

\begin{abstract}
Se estudió la efectividad del tiempo de acceso al pienso y la suplementación con alfalfa en pavos comerciales en finalización y en condiciones hipobáricas naturales. Se utilizó un arreglo factorial de $2 \times 2$ para evaluar los efectos principales de tiempo de acceso al pienso ( 12 y $24 \mathrm{~h} / \mathrm{d}$ ) y suplementación con alfalfa ( 0 y $1 \%$ del peso corporal). Adicionalmente se consideró el factor sexo para analizar los datos de características de carcasa, índices cardiacos y parámetros hematológicos. Un total de 256 pavos Hybrid Converter de 70 días de edad se asignaron a cuatro tratamientos, cada uno con cuatro repeticiones (repetición de 8 machos y 8 hembras en lote mixto). Todas las aves recibieron las mismas dietas durante los primeros 70 días de edad. Los tratamientos fueron $12 \mathrm{~h} /$ $\mathrm{d}-0 \%, 12 \mathrm{~h} / \mathrm{d}-1 \%, 24 \mathrm{~h} / \mathrm{d}-0 \%$ y $24 \mathrm{~h} / \mathrm{d}-1 \%$. A los 91 días de edad las aves fueron sacrificadas. No se observaron interacciones ni diferencias en los efectos principales para la
\end{abstract}

\footnotetext{
${ }^{1}$ Facultad de Ingeniería en Ciencias Pecuarias, Universidad Nacional de Cajamarca, Perú

${ }^{2}$ Laboratorio de Inmunología, Facultad de Ciencias Veterinarias, Universidad Nacional de Cajamarca, Perú

${ }^{3}$ E-mail: mparedes@unc.edu.pe
}

Recibido: 28 de marzo de 2021

Aceptado para publicación: 9 de octubre de 2021

Publicado: 22 de diciembre de 2021

CLos autores. Este artículo es publicado por la Rev Inv Vet Perú de la Facultad de Medicina Veterinaria, Universidad Nacional Mayor de San Marcos. Este es un artículo de acceso abierto, distribuido bajo los términos de la licencia Creative Commons Atribución 4.0 Internacional (CC BY 4.0) [https:// creativecommons.org/licenses/by/4.0/deed.es] que permite el uso, distribución y reproducción en cualquier medio, siempre que la obra original sea debidamente citada de su fuente original 
ingesta de alimento, pero hubo diferencia significativa en la ganancia de peso, con mayor incremento en pavos con acceso al pienso $12 \mathrm{~h} / \mathrm{d}$. La conversión alimenticia fue mejor en pavos con acceso al pienso durante $12 \mathrm{~h} / \mathrm{d}$ y que no consumieron alfalfa. La restricción de horas de acceso al pienso y la suplementación con alfalfa no afectaron el rendimiento de carcasa ni la acumulación de grasa abdominal. Se determinó que la suplementación con alfalfa evita agrandamientos degenerativos de corazón, ventrículo derecho e hígado, y contrarresta el estado de estrés del pavo ocasionado por la restricción del pienso.

Palabras clave: pavo, restricción de pienso, alfalfa, rendimiento productivo, parámetros hematológicos

\section{Abstract}

The effectiveness of feed access time and alfalfa supplementation in finishing commercial turkeys and under natural hypobaric conditions was studied. A $2 \times 2$ factorial arrangement of treatments was used to assess the main effects of time of access to feed $(12$ and $24 \mathrm{~h} / \mathrm{d})$ and supplementation with alfalfa ( 0 and $1 \%$ of body weight). Additionally, the sex factor was considered to analyse the data of carcass characteristics, cardiac indices and haematological parameters. A total of 256 70-day-old Hybrid Converter turkeys were assigned to four treatments, each with four replications ( 8 males and 8 females in mixed-flock). All birds received the same diets for the first 70 days of age. The treatments were $12 \mathrm{~h} / \mathrm{d}-0 \%, 12 \mathrm{~h} / \mathrm{d}-1 \%, 24 \mathrm{~h} / \mathrm{d}-0 \%$ and $24 \mathrm{~h} / \mathrm{d}-1 \%$. At 91 days of age the birds were slaughtered. No interactions or differences were observed in the main effects for feed intake, but there was a greater increase in body weigh in turkeys with access to feed $12 \mathrm{~h} / \mathrm{d}$. Feed conversion was better in turkeys with access to feed for $12 \mathrm{~h} / \mathrm{d}$ and that did not consume alfalfa. The restriction of hours of access to the feed and the supplementation with alfalfa did not affect the carcass performance or the accumulation of abdominal fat. Alfalfa supplementation was found to prevent degenerative enlargements of the heart, right ventricle, and liver, and counteract turkey stress caused by feed restriction.

Key words: turkey, feed restriction, alfalfa, productive performance, hematological parameters

\section{INTRODUCCIÓN}

En el Perú la producción anual de carne de pavo bordea las 30 mil toneladas (MINAGRI, 2020) a partir de pavos híbridos criados en granjas de alto nivel tecnológico, así como en pequeñas granjas. El pavo de rápido crecimiento, en cualquier sistema productivo y tamaño de granja, requiere cuidados básicos de bienestar animal que influyen sobre la productividad, evitando someterlo a condiciones desafiantes como galpón con alta densidad de aves, deficiente ventilación, cama húmeda o mala nutrición, lo cual provoca picaje (Marchewka et al., 2019), cojeras y mortalidad elevada (Ferrante et al., 2019). La mortalidad en pavos de engorde por lo general es alta, con mayor proporción de muertes en machos (Vasdal et al., 2021), especialmente por colibacilosis (Marrett et al., 2000), síndrome enteritis-mortalidad del pavipollo (Roy et al., 2002) y miocardiopatía dilatada (DCM), caracterizada por ventrículos agrandados (Fatkin y Graham, 2002). 
Se estima que la DCM presenta una mortalidad entre 2 y $4 \%$ y morbilidad reflejada en pérdida de peso (Zepeda y Kooyman, 2002). La etiología no es del todo conocida, pero los factores implicados en su incidencia y gravedad pueden ser genéticos o ambientales (Lin et al., 2006), como mala nutrición, manejo inadecuado, estrés (Poller et al., 2005). La DCM afecta a pavos de edad temprana y en fase de finalización, ocasionando muerte al momento de la captura con cuadros de insuficiencia cardíaca aguda y congestiva, agotamiento metabólico, deshidratación (Petracci et al., 2006). Asimismo, se observa incremento de la corticosterona plasmática de hasta $15 \mathrm{ng} / \mathrm{ml}$ y ratios heterófilos:linfocitos de 1.77 (Scanes et al., 2020).

Diversos tratamientos se han ensayado para controlar problemas entéricos en pavos a fin de evitar mortalidad. Se ha demostrado la eficacia del sulfato de neomicina contra $E$. coli (Marrett et al., 2000). La furazolidona es eficaz para tratar enteritis y diarreas, pero exacerba la DCM (Genao et al., 1996). El ácido propiónico dietario reduce la mortalidad del pavipollo por enteritis, sin disminuir el contenido de hemoglobina (Roy et al., 2002). Asimismo, el suplemento altamente digestible a base de $95.6 \%$ de maltodextrina en la fase de pre-sacrificio evita pérdida de peso y reduce la carga microbiana digestiva (Rathgeber et al., 2007). También se sugiere el uso de aceites funcionales en la dieta del pavo como una alternativa viable a los antibióticos promotores de crecimiento (Ferket et al., 2020) y que mayores niveles de metionina y arginina en la dieta favorecen el estado inmunológico del pavo (Jankowski et al., 2020). Por otro lado, densidades de crianza de $0.42 \mathrm{~m}^{2}$ por ave de 5 a 20 semanas de edad favorece la crianza de pavos más saludables y con mejor rendimiento (Bartz et al., 2020), en tanto que la crianza en condiciones hipobáricas naturales, el imidapril inhibe la remodelación ventricular derecha inducida por baja la temperatura ambiental (Hao et al., 2013).
Otras investigaciones apuntan a evaluar alternativas botánicas en la alimentación, así como la restricción alimenticia en aves de rápido crecimiento criadas en zonas frías e hipobáricas. La suplementación con concentrado proteico de alfalfa en pollos de engorde mejora las funciones metabólicas del organismo, la resistencia a procesos oxidativos y el perfil de ácidos grasos de la carne (Kwiecieñ et al., 2021). La alfalfa como suplemento de gallinas redujo las concentraciones de triglicéridos en sangre con relación a las alimentadas únicamente con pienso, además de contrarrestar el estrés fisiológico producido por la restricción de alimento durante la muda inducida (Dunkley et al., 2007). En el caso de pavos, la aplicación de la restricción alimenticia y el consecuente menor esfuerzo metabólico tiene beneficios en pavos en condiciones estresantes (Farghly et al., 2018).

Sin embargo, debe tenerse en cuenta que la restricción nutricional temprana afecta la respuesta y actividad de las células satélite, importantes en el crecimiento muscular (Velleman et al., 2019), retrasando la expresión de las isoformas de cadena de miosina pesada en los músculos pectoral y esquelético (Huffman et al., 2012). La restricción alimenticia de hasta $18 \mathrm{~h}$ de ayuno y a largo plazo, en pavos se asocia con disminución de la concentración de la hormona ghrelina (Vizcarra et al., 2018). Se ha evidenciado que gallinas reproductoras que ingieren alimento ad libitum pueden padecer hipertrofia cardíaca desadaptativa que progresa a una patogénesis en la contractilidad, lo cual podría evitarse con restricción alimenticia (Chen et al., 2017).

Pocos estudios han buscado delinear las relaciones entre indicadores biológicos y la alimentación del pavo de engorde en fase de finalización que es cuando el ave tiene mayor incremento de masa corporal y las alteraciones metabólicas pueden comprometer la función cardíaca. El presente estudio tuvo como objetivo evaluar el desempeño produc- 
tivo, características de la carcasa, indicadores cardiacos y hematológicos de pavos en fase de finalización con consumos de pienso restringido y ad libitum, suplementados con alfalfa fresca, en condición hipobárica natural de los Andes peruanos.

\section{Materiales y Métodos}

\section{Aves, Pienso y Diseño Experimental}

Se trabajó con 256 pavos de engorde, 128 machos y 128 hembras, de la línea Hybrid Converter de 70 días de edad, criados en la granja avícola experimental de la Facultad de Ingeniería en Ciencias Pecuarias de la Universidad Nacional de Cajamarca, Perú. Las aves estuvieron alojadas en un galpón dividido en 16 corrales de $8 \mathrm{~m}^{2}$ hasta los 91 días de edad. Los pavos fueron pesados al inicio del estudio. El alimento, en forma de harina, fue suministrado de acuerdo con el tratamiento asignado y el agua fue provista ad libitum. La fórmula alimenticia del pienso de finalización y el contenido nutricional estimado se presentan en el Cuadro 1.

Los pavos fueron distribuidos en cuatro grupos con cuatro corrales por tratamiento. Cada corral con 16 pavos ( 8 machos y 8 hembras) fue considerado como una unidad experimental o repetición. Todas las aves recibieron el mismo pienso, pero variando las horas de acceso a este $(12$ y $24 \mathrm{~h} / \mathrm{d})$ y los niveles de suplementación con alfalfa fresca $(0$ y $1 \%)$. Los tratamientos fueron: $12 \mathrm{~h} / \mathrm{d}-$ $0 \%, 12 \mathrm{~h} / \mathrm{d}-1 \%, 24 \mathrm{~h} / \mathrm{d}-0 \%, 24 \mathrm{~h} / \mathrm{d}-1 \%$.

\section{Suministro de Alfalfa}

La alfalfa fue cosechada diariamente de un predio agrícola ubicado en el sector Huacariz del valle Cajamarca. El forraje fue picado manualmente con la ayuda de un machete y sobre una base de madera, considerando una longitud de corte del tallo entre 1$2 \mathrm{~cm}$ y suministrado en comedero de plástico, tipo tolva y exclusivo para el forraje, pre-
Cuadro 1. Ingredientes $\mathrm{y}$ contenido nutricional del pienso $(\mathrm{g} / \mathrm{kg}$, base fresca) utilizado en el experimento

\begin{tabular}{|c|c|}
\hline & $\mathrm{g} / \mathrm{kg}$ \\
\hline \multicolumn{2}{|l|}{ Ingredientes } \\
\hline Maíz amarillo & 595 \\
\hline Torta de soya & 300 \\
\hline Harina de pescado & 15 \\
\hline Aceite de palma & 50 \\
\hline Fosfato dicálcico & 20 \\
\hline Carbonato de calcio & 9 \\
\hline DL Metionina & 1 \\
\hline Sal común, $\mathrm{NaCl}$ & 4 \\
\hline Premezcla vit/minerales ${ }^{1}$ & 1 \\
\hline Cloruro de colina $60 \%$ & 1 \\
\hline Lisina $\mathrm{HCl}$ & 3 \\
\hline L Treonina & 1 \\
\hline \multicolumn{2}{|l|}{ Contenido nutricional calculado } \\
\hline Materia seca & 885.4 \\
\hline Proteína cruda & 214.7 \\
\hline Energía metabolizable, $\mathrm{kcal} / \mathrm{kg}$ & 3312 \\
\hline Grasa & 65.1 \\
\hline Metionina & 6.4 \\
\hline Lisina & 16.2 \\
\hline Treonina & 9.3 \\
\hline Triptófano & 2.6 \\
\hline $\mathrm{Ca}$ & 11.2 \\
\hline P disponible & 6.5 \\
\hline $\mathrm{Na}$ & 1.7 \\
\hline \multicolumn{2}{|c|}{$\begin{array}{l}{ }^{1} \text { Cada kg contiene: Vit. A } 10000 \text { mil UI, Vit. D3 } 3 \\
000 \text { mil UI, Vit. E } 12000 \mathrm{Ul} \text {, Vit. K3 } 2.5 \mathrm{~g} \text {, tiamina } \\
2 \mathrm{~g} \text {, riboflavina } 6 \mathrm{~g} \text {, cianocobalamina } 12 \mathrm{mg} \text {, } \\
\text { ácido pantoténico } 16 \mathrm{~g} \text {, ácido fólico } 21,5 \mathrm{~g} \text {, } \\
\text { niacina } 120 \mathrm{mg}, \mathrm{Mn} 65 \mathrm{~g}, \mathrm{Zn} 65 \mathrm{~g} \text {, Fe } 80 \mathrm{~g} \text {, Cu } 10 \\
\mathrm{~g}, 11 \mathrm{~g} \text {, Se } 200 \mathrm{mg} \text {. Producto comercializado } \\
\text { como Proapack Pavos por Distribuidora Montana } \\
\text { S.A., Perú }\end{array}$} \\
\hline
\end{tabular}

vio pesado y estimación de las cantidades de alfalfa para cada corral. Cada tres días se determinó el contenido de materia seca (MS) de la alfalfa en la estufa a $105^{\circ} \mathrm{C} \times 24 \mathrm{~h}$, resultando en $23.8 \%$. La cantidad de alfalfa 
asignada a cada tratamiento y corral se calculó según el peso corporal de los pavos registrados al inicio de cada semana y corregida al inicio de la siguiente. La alfalfa se suministró diariamente a las 09:00 $\mathrm{h}$ en los ocho corrales suplementados con forraje.

\section{Rendimiento Productivo}

El peso corporal (PC) e ingesta de alimento por corral fueron determinados en una balanza electrónica de plataforma TCS de $100 \mathrm{~kg}$ de capacidad y precisión de $10 \mathrm{~g}$. Los datos se registraron semanalmente. Se determinó la ingesta de alimento promedio por ave para todo el periodo experimental, para lo cual se estimó el consumo de alimento según tratamiento considerando el consumo de concentrado y de alfalfa en términos de materia seca (MS). El índice de conversión alimenticia (ICA) fue determinado por la relación consumo de MS/incremento de PC.

A la edad de 91 días, 4 pavos por corral ( 2 machos y 2 hembras) fueron elegidos al azar, aturdidos eléctricamente y sacrificados previo ayuno de $8 \mathrm{~h}$. La carcasa, hígado, molleja, intestinos y grasa abdominal se pesaron en balanza de precisión de $0.01 \mathrm{~g}$. Fue considerada como carcasa todo el cuerpo del animal desprovisto de plumas desde la cabeza, cuello, alas, patas, así como hígado, corazón y molleja lavada, considerando el criterio comercial que demanda la carcasa del pavo navideño.

Los pesos relativos de carcasa, grasa abdominal y órganos digestivos fueron determinados en base al peso absoluto expresados en relación al peso vivo del ave (\%). Por lo tanto, se determinó el rendimiento de carcasa (RC) mediante la siguiente fórmula: $\mathrm{RC}=$ (peso de carcasa/peso vivo final) $\times 100$; grasa abdominal $=($ peso de la grasa abdominal de la carcasa/peso vivo final) x 100; Molleja $=($ peso molleja/peso vivo final $) \times 100$; Intestinos $=($ peso intestinos $/$ peso vivo final $)$ $\mathrm{x} 100$; Hígado $=$ (peso hígado/peso vivo final) x 100 .

\section{Índices Cardiacos y Parámetros Hema- tológicos}

Se colectaron los corazones para obtener los pesos ventriculares. Se pesaron los corazones y luego se retiró la grasa pericárdica, las aurículas y las válvulas cardíacas. Se pesaron los ventrículos y determinó la relación peso del ventrículo derecho dividido entre el peso de la masa ventricular total (IVD/VT). Las ecuaciones utilizadas fueron las siguientes: peso relativo del corazón (\%) $=($ peso de corazón/peso vivo final $) \times 100 ;$ I$\mathrm{VD} / \mathrm{VT}=$ peso del ventrículo derecho/peso de los ventrículos derecho e izquierdo.

El análisis hematológico se realizó en el laboratorio de inmunología veterinaria de la Universidad Nacional de Cajamarca. Se recolectaron muestras de sangre mediante venopunción de la vena braquial de un pavo macho y una hembra de cada corral a los 91 días de edad en tubos Vacutainer de $5 \mathrm{ml}$ con EDTA. El análisis del número de glóbulos rojos $\left(\mathrm{GR}, 10^{3} / \mu 1\right)$, la concentración de hemoglobina en sangre $(\mathrm{HG}, \mathrm{g} / \mathrm{dl})$ y el hematocrito (HT, \%) se realizó utilizando el analizador de hematología laser Hemavet 950. El conteo de glóbulos blancos y las proporciones (porcentaje) de heterófilos y linfocitos se determinaron con un sistema de análisis de sangre que utiliza luz láser. El indicador de estrés ratio heterófilos/linfocitos (RHL) se calculó dividiendo el número de heterófilos en $1 \mathrm{ml}$ de sangre periférica por el número de linfocitos.

\section{Análisis Estadístico}

Los datos del experimento se sometieron a análisis estadístico utilizando análisis de varianza del procedimiento GLM del SAS (SAS, 2003). Los datos de rendimiento productivo se analizaron según arreglo factorial $2 \times 2$ para evaluar los efectos principales del tiempo de acceso al pienso y la suplementación con alfalfa y sus interacciones. Los datos de rendimiento de carcasa, índices cardiacos y valores hematológicos se analizaron en arreglo factorial de 2 × 2 × 2 para 
evaluar los efectos principales de sexo, tiempo de acceso al pienso y la suplementación con alfalfa. Se comprobaron los datos de todos los indicadores evaluados para distribución normal.

En los indicadores de rendimiento productivo un corral constituyó una unidad experimental. En los indicadores de rendimiento de carcasa e índices cardiacos, los datos de dos aves representaron una repetición. En los parámetros hematológicos los valores de un ave representaron una unidad experimental. Los valores presentados en las tablas son medias con error estándar agrupado de la media (SEM). Al detectarse un efecto significativo, las diferencias entre tratamientos o efectos principales se analizaron por prueba de rango múltiple de Duncan.

\section{Resultados y Discusión}

\section{Rendimiento Productivo}

La ingesta de alimento, ganancia de peso e índice de conversión alimenticia de pavos de 11 a 13 semanas de edad con diferente tiempo de acceso al pienso y suplementados con alfalfa fresca se muestran en el Cuadro 2. No se encontraron diferencias $(p>0.05)$ entre tratamientos ni en los efectos principales para la ingesta de alimento, pero hubo diferencias significativas $(p<0.05)$ en la ganancia de peso e ICA, observándose mayor incremento de peso en los pavos con acceso al pienso $12 \mathrm{~h} / \mathrm{d}$. La conversión alimenticia fue mejor en pavos con acceso al pienso durante $12 \mathrm{~h} / \mathrm{d}$ y que no consumieron alfalfa, seguidos por los pavos con acceso al pienso durante $12 \mathrm{~h} / \mathrm{d}$ y suplementados con alfalfa. El ICA más pobre correspondió a los pavos con acceso al pienso durante $24 \mathrm{~h} / \mathrm{d}$, reflejado también en los efectos principales, en los que se observa un mejor ICA en pavos con acceso al pienso de $12 \mathrm{~h} / \mathrm{d}$ en comparación a $24 \mathrm{~h} /$ $\mathrm{d}$ durante la fase de finalización. También se encontró que la suplementación con alfalfa del pavo afecta negativamente el indicador de eficiencia alimenticia.
El mayor tiempo de acceso al pienso de los pavos en finalización generó menor incremento de peso corporal y una conversión alimenticia menos eficiente, debido posiblemente a que ingestas ad libitum de pienso provocan mayor esfuerzo digestivo y trabajo metabólico, lo cual puede afectar el funcionamiento cardiaco de las aves (Chen et al., 2017), sobre todo en condiciones hipobáricas naturales. Por otro lado, la suplementación con alfalfa disminuyó la eficiencia alimenticia, aunque no se observaron diferencias en la ganancia de peso entre los pavos que consumieron alfalfa y los que fueron privados de la ingesta del forraje. Posiblemente el mayor consumo de fibra de los pavos con ingestas de alfalfa pudo haber generado menor eficiencia alimenticia, por ser la fibra uno de los principales componentes químicos del alimento que afectan la digestibilidad ileal de los nutrientes en aves (Singh et al., 2019). El ICA de los pavos estuvo por debajo del ICA reportado por Jankowski et al. (2020) en pavos en fase alimenticia similar, pero criados a nivel del mar, con el uso único de pienso y sin suplementación de alfalfa; condiciones que pudieron influenciar en este indicador, por cuanto, las condiciones ambientales en las que se crían las aves están asociadas directamente con el peso corporal e ICA de los pavos (Bartz et al., 2020).

\section{Características de Carcasa}

En el Cuadro 3 se observan los valores promedio del rendimiento de carcasa, pesos relativos de la grasa abdominal, molleja, intestinos e hígado de los pavos. No se observaron diferencias $(p>0.05)$ entre las medias de los tratamientos y efectos principales para el rendimiento de carcasa, pero hubo interacciones entre los niveles de los tres factores evaluados para los indicadores grasa abdominal, molleja, intestinos e hígado. Mayor cantidad de grasa abdominal se encontró en las hembras que en los machos, la molleja tuvo mayor peso en los pavos que tuvieron acceso al pienso durante $24 \mathrm{~h} / \mathrm{d}$. El hígado alcanzó mayor tamaño en las aves con acceso restringido al pienso y sin suplementación de alfalfa. 
Cuadro 2. Ingesta de alimento, ganancia de peso e índice de conversión alimenticia (ICA) de pavos de 11 a 13 semanas de edad ${ }^{1}$ con diferente tiempo de acceso al pienso y suplementados con alfalfa ${ }^{2}$

\begin{tabular}{|c|c|c|c|}
\hline & $\begin{array}{l}\text { Ingesta de alimento } \\
\text { (kg de MS) }\end{array}$ & $\begin{array}{c}\text { Ganancia de peso } \\
(\mathrm{kg})\end{array}$ & $\begin{array}{c}\text { ICA } \\
\left(\mathrm{kg} \text { de } \mathrm{MS} \cdot \mathrm{kg}^{-1}\right) \\
\end{array}$ \\
\hline \multicolumn{4}{|l|}{ Tratamientos } \\
\hline \multicolumn{4}{|l|}{ Pienso x Alfalfa } \\
\hline $12 \mathrm{~h} / \mathrm{d} \times 0 \%$ & 8.78 & $3.58^{\mathrm{a}}$ & $2.45^{\mathrm{d}}$ \\
\hline $12 \mathrm{~h} / \mathrm{d} \times 1 \%$ & 8.89 & $3.52^{\mathrm{a}}$ & $2.53^{\mathrm{c}}$ \\
\hline $24 \mathrm{~h} / \mathrm{d} \times 0 \%$ & 8.65 & $3.32^{\mathrm{b}}$ & $2.60^{\mathrm{b}}$ \\
\hline $24 \mathrm{~h} / \mathrm{d} \times 1 \%$ & 8.79 & $3.31^{\mathrm{b}}$ & $2.65^{\mathrm{a}}$ \\
\hline SEM & 0.049 & 0.069 & 0.043 \\
\hline \multicolumn{4}{|l|}{ Efectos principales } \\
\hline \multicolumn{4}{|l|}{ Pienso } \\
\hline $12 \mathrm{~h} / \mathrm{d}$ & 8.83 & $3.55^{\mathrm{a}}$ & $2.49^{\mathrm{b}}$ \\
\hline $24 \mathrm{~h} / \mathrm{d}$ & 8.70 & $3.32^{\mathrm{b}}$ & $2.62^{\mathrm{a}}$ \\
\hline \multicolumn{4}{|l|}{ Alfalfa } \\
\hline $0 \%$ & 8.71 & 3.45 & $2.52^{\mathrm{b}}$ \\
\hline $1 \%$ & 8.83 & 3.42 & $2.58^{\mathrm{a}}$ \\
\hline \multicolumn{4}{|l|}{$\mathrm{p}$-value } \\
\hline Pienso & 0.309 & 0.027 & 0.042 \\
\hline Alfalfa & 0.461 & 0.064 & 0.046 \\
\hline Pienso x alfalfa & 0.731 & 0.033 & 0.027 \\
\hline
\end{tabular}

${ }^{1}$ Cada valor representa la media de cuatro repeticiones para los efectos del tratamiento y ocho repeticiones para los efectos principales. Cada repetición estuvo conformada por 16 pavos en lote mixto (8 hembras y 8 machos)

${ }^{2}$ Los niveles de suplementación con alfalfa fresca fueron calculados en relación al peso corporal del pavo

SEM: Error estándar de la media

$a, b, c, d$ Las medias dentro de una columna que no comparten igual superíndice difieren significativamente $(p<0.05)$ para los efectos del tratamiento y para los efectos principales

Los valores de rendimiento de carcasa no estuvieron influenciados por el programa de alimentación a diferencia de los pesos de molleja, hígado y corazón, coincidiendo con los resultados reportados por Laudadio et al. (2009) en pavos de 16 semanas. Estas similitudes pueden deberse a la poca diferencia nutricional entre tratamientos, que no generaron mayores cambios en rendimientos de canal. El mayor peso del hígado pudo estar influenciado por la alimentación única de pienso sin ingesta de alfalfa, antes que, por la restricción alimentaria, en contraste a lo encontrado por Chen et al. (2017). Los pesos relativos de molleja e intestinos no se vieron afectados por la inclusión de alfalfa en la dieta del pavo como hipotéticamente se había proyectado. 
Cuadro 3. Rendimiento de carcasa (RC), peso relativo de órganos digestivos y de la grasa abdominal de pavos de 13 semanas de edad ${ }^{1}$ alimentados con diferente tiempo de acceso al pienso y suplementados con alfalfa ${ }^{2}$ en fase de finalización

\begin{tabular}{|c|c|c|c|c|c|}
\hline & $\mathrm{RC}(\%)$ & $\begin{array}{c}\text { Grasa } \\
\text { abdominal } \\
(\%)\end{array}$ & $\begin{array}{c}\text { Molleja } \\
(\%)\end{array}$ & $\begin{array}{c}\text { Intestinos } \\
(\%)\end{array}$ & $\begin{array}{c}\text { Hígado } \\
(\%)\end{array}$ \\
\hline \multicolumn{6}{|l|}{ Tratamientos } \\
\hline \multicolumn{6}{|l|}{ Sexo x Pienso x Alfalfa } \\
\hline Macho x 12 h/d x 0\% & 86.61 & $1.09^{\mathrm{b}}$ & $1.55^{\mathrm{c}}$ & $4.33^{\mathrm{a}}$ & $1.36^{\mathrm{b}}$ \\
\hline Macho x $12 \mathrm{~h} / \mathrm{d} \times 1 \%$ & 84.61 & $0.85^{\mathrm{c}}$ & $1.59^{\mathrm{c}}$ & $4.01^{\mathrm{b}}$ & $1.23^{\mathrm{c}}$ \\
\hline Macho x 24 h/d x 0\% & 86.49 & $0.95^{\mathrm{c}}$ & $1.53^{\mathrm{c}}$ & $3.73^{\mathrm{c}}$ & $1.27^{\mathrm{c}}$ \\
\hline Macho x 24 h/d x 1\% & 86.34 & $0.77^{\mathrm{c}}$ & $1.69^{\mathrm{c}}$ & $4.00^{\mathrm{b}}$ & $1.30^{\mathrm{c}}$ \\
\hline Hembra $\times 12 \mathrm{~h} / \mathrm{d} \times 0 \%$ & 85.79 & $1.12^{\mathrm{b}}$ & $1.84^{\mathrm{b}}$ & $3.83^{\mathrm{c}}$ & $1.81^{\mathrm{a}}$ \\
\hline Hembra x $12 \mathrm{~h} / \mathrm{d} \times 1 \%$ & 83.85 & $1.18^{\mathrm{b}}$ & $1.84^{\mathrm{b}}$ & $3.61^{\mathrm{c}}$ & $1.14^{\mathrm{a}}$ \\
\hline Hembra $\times 24 \mathrm{~h} / \mathrm{d} \times 0 \%$ & 85.56 & $1.14^{\mathrm{b}}$ & $2.27^{\mathrm{a}}$ & $3.26^{\mathrm{d}}$ & $1.42^{\mathrm{b}}$ \\
\hline Hembra $\times 24 \mathrm{~h} / \mathrm{d} \times 1 \%$ & 85.29 & $1.27^{\mathrm{a}}$ & $2.23^{\mathrm{a}}$ & $3.59^{\mathrm{c}}$ & $1.14^{\mathrm{d}}$ \\
\hline SEM & 0.341 & 0.007 & 0.103 & 0.115 & 0.081 \\
\hline \multicolumn{6}{|l|}{ Efectos principales } \\
\hline \multicolumn{6}{|l|}{ Sexo } \\
\hline Macho & 86.01 & $0.91^{\mathrm{b}}$ & $1.59^{\mathrm{b}}$ & $4.02^{\mathrm{a}}$ & $1.29^{\mathrm{b}}$ \\
\hline Hembra & 85.12 & $1.18^{\mathrm{a}}$ & $2.04^{\mathrm{a}}$ & $3.57^{\mathrm{b}}$ & $1.36^{\mathrm{a}}$ \\
\hline \multicolumn{6}{|l|}{ Pienso } \\
\hline $12 \mathrm{~h} / \mathrm{d}$ & 85.21 & 1.06 & $1.70^{\mathrm{b}}$ & 3.94 & $1.36^{\mathrm{a}}$ \\
\hline $24 \mathrm{~h} / \mathrm{d}$ & 85.92 & 1.03 & $1.93^{\mathrm{a}}$ & 3.64 & $1.28^{\mathrm{b}}$ \\
\hline \multicolumn{6}{|l|}{ Alfalfa } \\
\hline $0 \%$ & 86.11 & 1.07 & 1.80 & 3.79 & $1.46^{\mathrm{a}}$ \\
\hline $1 \%$ & 85.02 & 1.02 & 1.84 & 3.80 & $1.18^{\mathrm{b}}$ \\
\hline \multicolumn{6}{|l|}{ p-value } \\
\hline Sexo x Pienso x Alfalfa & 0.738 & 0.025 & 0.018 & 0.027 & 0.038 \\
\hline Sexo x Pienso & 0.126 & 0.093 & 0.063 & 0.241 & 0.131 \\
\hline Sexo x Alfalfa & 0.094 & 0.391 & 0.261 & 0.186 & 0.078 \\
\hline Pienso x Alfalfa & 0.285 & 0.628 & 0.085 & 0.246 & 0.251 \\
\hline Sexo & 0.073 & 0.034 & 0.043 & 0.034 & 0.045 \\
\hline Pienso & 0.151 & 0.261 & 0.047 & 0.274 & 0.049 \\
\hline Alfalfa & 0.539 & 0.142 & 0.483 & 0.769 & 0.016 \\
\hline
\end{tabular}

1 Cada valor representa la media de cuatro repeticiones para los efectos del tratamiento y 16 repeticiones para los efectos principales. Cada repetición estuvo conformada por 2 pavos

2 Los niveles de suplementación con alfalfa fresca fueron calculados en relación al peso corporal del pavo

SEM: Error estándar de la media

$a, b, c, d$ Las medias dentro de una columna que no comparten igual superíndice difieren significativamente $(p<0.05)$ para los efectos del tratamiento y para los efectos principales

$\mathrm{RC}=($ Peso de carcasa/Peso vivo final $) \times 100 ;$ Grasa abdominal $(\%)=($ Peso de la grasa abdominal de la carcasa/Peso vivo final) $\times 100$; Molleja $(\%)=($ Peso molleja/Peso vivo final $) \times 100 ;$ Intestinos $(\%)=($ Peso intestinos/Peso vivo final) $\times 100$; Hígado $(\%)=($ Peso hígado/Peso vivo final) $\times 100$ 
Cuadro 4. Peso relativo de corazón e índice ventrículo derecho/ventrículo total (I-VD/VT) de pavos de 13 semanas de edad ${ }^{1}$ alimentados con diferente tiempo de acceso al pienso y suplementados con alfalfa ${ }^{2}$ en fase de finalización

\begin{tabular}{|c|c|c|}
\hline & Corazón $(\%)$ & $\mathrm{I}-\mathrm{VD} / \mathrm{VT}$ \\
\hline \multicolumn{3}{|l|}{ Tratamientos } \\
\hline \multicolumn{3}{|l|}{ Sexo x Pienso x Alfalfa } \\
\hline Macho x $12 \mathrm{~h} / \mathrm{d}$ x 0\% & $0.43^{\mathrm{c}}$ & $0.26^{\mathrm{a}}$ \\
\hline Macho x $12 \mathrm{~h} / \mathrm{d}$ x $1 \%$ & $0.58^{\mathrm{a}}$ & $0.21^{\mathrm{b}}$ \\
\hline Macho x 24 h/d x 0\% & $0.61^{\mathrm{a}}$ & $0.25^{\mathrm{a}}$ \\
\hline Macho x 24 h/d x 1\% & $0.62^{\mathrm{a}}$ & $0.23^{b}$ \\
\hline Hembra $\times 12$ h/d x $0 \%$ & $0.54^{\mathrm{b}}$ & $0.19^{\mathrm{c}}$ \\
\hline Hembra $\times 12 \mathrm{~h} / \mathrm{d} \times 1 \%$ & $0.38^{\mathrm{c}}$ & $0.18^{\mathrm{c}}$ \\
\hline Hembra $\times 24 \mathrm{~h} / \mathrm{d} \times 0 \%$ & $0.57^{\mathrm{a}}$ & $0.23^{\mathrm{b}}$ \\
\hline Hembra $\times 24 \mathrm{~h} / \mathrm{d} \times 1 \%$ & $0.39^{\mathrm{c}}$ & $0.22^{\mathrm{b}}$ \\
\hline SEM & 0.035 & 0.010 \\
\hline \multicolumn{3}{|l|}{ Efectos principales } \\
\hline \multicolumn{3}{|l|}{ Sexo } \\
\hline Macho & $0.56^{\mathrm{a}}$ & $0.24^{\mathrm{a}}$ \\
\hline Hembra & $0.47^{\mathrm{b}}$ & $0.20^{\mathrm{b}}$ \\
\hline \multicolumn{3}{|l|}{ Pienso } \\
\hline $12 \mathrm{~h} / \mathrm{d}$ & $0.48^{\mathrm{b}}$ & $0.21^{\mathrm{b}}$ \\
\hline $24 \mathrm{~h} / \mathrm{d}$ & $0.55^{\mathrm{a}}$ & $0.23^{\mathrm{a}}$ \\
\hline \multicolumn{3}{|l|}{ Alfalfa } \\
\hline $0 \%$ & $0.54^{\mathrm{a}}$ & $0.23^{\mathrm{a}}$ \\
\hline $1 \%$ & $0.49^{\mathrm{b}}$ & $0.21^{\mathrm{b}}$ \\
\hline \multicolumn{3}{|l|}{ p-value } \\
\hline Sexo x Pienso x Alfalfa & 0.017 & 0.048 \\
\hline Sexo x Pienso & 0.021 & 0.093 \\
\hline Sexo x Alfalfa & 0.039 & 0.073 \\
\hline Pienso x Alfalfa & 0.027 & 0.131 \\
\hline Sexo & 0.009 & 0.025 \\
\hline Pienso & 0.042 & 0.047 \\
\hline Alfalfa & 0.046 & 0.043 \\
\hline
\end{tabular}

${ }^{1}$ Cada valor representa la media de cuatro repeticiones para los efectos del tratamiento y 16 repeticiones para los efectos principales. Cada repetición estuvo conformada por 2 pavos

2 Los niveles de suplementación con alfalfa fresca fueron calculados en relación al peso corporal del pavo.

SEM: Error estándar de la media

$a, b, c, d$ Las medias dentro de una columna que no comparten igual superíndice difieren significativamente $(p<0.05)$ para los efectos del tratamiento y para los efectos principales

Corazón $(\%)=($ Peso de corazón/Peso vivo final) $\times 100 ; \mathrm{I}-\mathrm{VD} / \mathrm{VT}=$ Peso del ventrículo derecho/Peso de los ventrículos derecho e izquierdo 


\section{Índices Cardiacos}

El Cuadro 4 muestra los pesos relativos de corazón e índice ventrículo derecho/ ventrículo total (I-VD/VT) de los pavos sacrificados a las 13 semanas de edad. Se observan las interacciones entre los tres factores en estudio y los efectos principales $(p<0.05)$. Los machos con acceso al pienso de $12 \mathrm{~h} / \mathrm{d}$ sin ingesta de alfalfa mostraron menor tamaño de corazón al igual que las hembras suplementadas con alfalfa que tuvieron acceso al pienso 12 y $24 \mathrm{~h} / \mathrm{d}$. El mayor peso relativo de corazón lo alcanzó el tratamiento de pavos machos con acceso al pienso las $24 \mathrm{~h} / \mathrm{d}$ sin tener en cuenta la suplementación con alfalfa, seguido de las hembras con acceso al pienso las $24 \mathrm{~h} / \mathrm{d}$ que no consumieron alfalfa. Al observar los promedios de los tres factores principales, el peso relativo del corazón está influenciado por los tres factores evaluados, siendo mayor en machos que en hembras, mayor en pavos con acceso al pienso durante $24 \mathrm{~h}$ que con $12 \mathrm{~h} / \mathrm{d}$ y menor en pavos que consumieron alfalfa. Esta respuesta también es similar en el I-VD/VT.

Estos hallazgos podrían sugerir prácticas alimenticias como el acceso al pienso de $12 \mathrm{~h} / \mathrm{d}$ y suministro de alfalfa en la última fase de crecimiento del pavo a fin de reducir trastornos cardiacos, que según investigaciones en esta especie a la edad de mercado y en condiciones tecnológicas de crianza óptima e industrial puede superar 2.9\% (Vecerek et al., 2019). Al analizar los indicadores cardiacos se debe considerar que el incremento de la masa de tejido cardiaco en el pavo de engorde está ligado a DCM con aumento de tamaño del ventrículo derecho, hipoxemia, viscosidad de la sangre, debilidad del músculo cardíaco y aparición de insuficiencia cardíaca (Frame et al., 2001).

La hipertrofia ventricular derecha es una respuesta al aumento de trabajo cardiaco y puede estar asociada con la ingesta de alimento, tal como sucede en el pollo de engorde ascítico (Kamely et al., 2015). Esta hiper- trofia en el presente estudio fue valorada por el I-VD/VT, que cuando es numéricamente elevado produce mermas o supresión del crecimiento y es causa de mortalidad (Balog, 2003). En este experimento, los valores altos de I-VD/VT, aparentemente correlacionados con mayor peso relativo del corazón, representaron indicios de casos de DCM asociada con menor ganancia de peso. Los pavos con acceso al pienso durante $24 \mathrm{~h} / \mathrm{d}$ tuvieron un menor incremento de peso que los pavos con acceso al pienso de $12 \mathrm{~h} / \mathrm{d}$ (3.32 vs. $3.55 \mathrm{~kg}$ ), mostrando los pavos con menor ganancia de peso un corazón más agrandado $(0.55 \% \mathrm{del}$ peso corporal vs. $0.48 \%$ ) y del mismo modo mayor I-VD/VT (0.23 vs. 0.21 ). Evidencias claras que el mayor trabajo cardiaco del pavo derivado del posible acceso continuo al pienso en las últimas tres semanas de engorde afecta negativamente el incremento de peso y la eficiencia alimenticia. Por otro lado, se demuestra que la ingesta de alfalfa en el pavo evita el agrandamiento del corazón y del ventrículo derecho.

Las hembras con acceso al pienso de $12 \mathrm{~h} / \mathrm{d}$ y suplementados con alfalfa mostraron menores indicios fisiológicos de DCM, con menores valores de I-VD/VT, que los pavos machos con acceso al pienso durante $24 \mathrm{~h} / \mathrm{d}$ y sin consumo de alfalfa. Sin embargo, de acuerdo con el valor de I-VD/VT reportado por Terry et al. (2012) para pavos comerciales de dos líneas genéticas, este varía entre 0.15 y 0.16 en condiciones de crianza a nivel del mar; por lo que podría notarse el efecto de un factor no evaluado en el presente estudio como es la altitud sobre el nivel del mar, que estaría provocando mayor desarrollo del tejido cardiaco ante una menor presión de oxígeno ambiental (Peñaloza y Arias, 2011). En la presente investigación los valores de I-VD/VT no significaron causa de muerte antes del sacrificio de los pavos de engorde, por lo que se puede concluir que el I-VD/VT $<0.23$ en promedio en el pavo híbrido no genera muerte, con valores de I$\mathrm{VD} / \mathrm{VT}$ de hasta 0.26 encontrado en algunos individuos aparentemente sanos a pesar de observarse ventrículo derecho hipertrófico. 


\section{Parámetros Hematológicos}

En el Cuadro 5 se indican los parámetros hematológicos. Se observan interacciones $(p<0.05)$ entre los factores evaluados en los parámetros hematocrito y RHL. Los pavos machos con acceso al pienso $24 \mathrm{~h} / \mathrm{d}$ exhibieron valores altos de HT, lo que sugiere una mayor capacidad de transporte de oxígeno en la sangre (Özkan et al., 2010). Es probable que se trate de una respuesta fisiológica adaptativa a la baja disponibilidad de oxígeno debido al mayor esfuerzo metabólico en condiciones hipobáricas (Beker et al., 2003). Del mismo modo, los valores de HT coinciden con los recuentos altos de GR y niveles altos de hemoglobina, ambas respuestas compensatorias a la baja presión atmosférica de oxígeno. El HT elevado está asociado a mayor viscosidad de la sangre, y en condiciones de presión atmosférica baja de oxígeno genera constricción de los vasos sanguíneos pulmonares, aumentando resistencia vascular pulmonar, lo que conduce a hipertensión pulmonar (Wideman, 2000).

Se debe considerar que los pavos fueron criados durante las cinco primeras semanas en ambiente controlado en cuanto a temperatura, quedando posteriormente expuestos al microclima natural que se genera dentro del galpón e influenciado por las bajas temperaturas ambientales que se tienen fuera de la caseta y la mayor producción de $\mathrm{CO}_{2} \mathrm{y}$ amoniaco en el interior. Tal cambio debería conducir a un incremento sustancial de HT debido a una mayor producción de eritrocitos (Yahav et al., (1997); sin embargo, se observa que, en tales circunstancias, la cantidad de eritrocitos tuvo relación con el contenido de hemoglobina, sin encontrarse interacciones entre los factores evaluados, excepto en el HT, lo que dio lugar la posibilidad en algunos pavos de mejorar la capacidad de transporte de oxígeno de la sangre, aumentando la viscosidad y con indicios de DCM (Luger et al., (2003). En el presente estudio, se pudo observar mayor presencia de HT en pavos machos que no consumieron alfalfa, pero no existe relación entre algún posible componen- te químico de la alfalfa con este parámetro hematológico, aun cuando la alfalfa puede tener propiedades de disminuir colesterol y triglicéridos en sangre (Dunkley et al., 2007). Un aspecto evidenciable en el presente trabajo resulta ser la posible correlación positiva entre la masa corporal y HT, HG y GR, tal como lo reporta González-Quintanilla et al. (2014). Por lo tanto, parece que el alto HT resultante observado en los pavos machos que no consumieron alfalfa está relacionado con el mayor peso corporal y mayor trabajo cardiaco, a fin de equilibrar la demanda y el suministro de oxígeno.

En el presente estudio, los pavos que tuvieron acceso restringido al pienso tuvieron una reacción más severa al estrés que los pavos con acceso al pienso las $24 \mathrm{~h}$, como lo demuestra el aumento de los RHL (2.06 vs. 1.36). Los RHL son una medida reconocida de estrés en las aves (Al-Murrani et al., 2002), que guardan relación con los niveles de corticosterona (Vizcarra et al., 2018) y se ha convertido en una valiosa herramienta para investigaciones sobre estrés. Los valores de RHL hallados en el presente estudio coinciden con los rangos de RHL encontrados en pavos comerciales bajo condiciones similares de crianza, pudiendo subir estos valores hasta 6 en casos de enfermedad (Huff et al., 2005). Se puede inferir que la restricción de horas de acceso al pienso en el pavo genera estrés; sin embargo, esta situación estresora se puede contrarrestar con el suministro de alfalfa, demostrado en el presente estudio por la disminución del RHL de 1.98 a 1.45.

\section{Conclusiones}

- La restricción de acceso al pienso de 12 horas por día en el pavo de 11 a 13 semanas de edad le permite alcanzar mayor ganancia de peso y mejor conversión alimenticia con relación a cuando tiene acceso al pienso $24 \mathrm{~h} / \mathrm{d}$.

- La suplementación con alfalfa fresca del pavo evita el agrandamiento degenerativo del corazón, ventrículo derecho e 
Cuadro 5. Parámetros hematológicos y ratio heterófilos/linfocitos (RHL) de pavos de 13 semanas de edad ${ }^{1}$ alimentados con diferente tiempo de acceso al pienso y suplementados con alfalfa ${ }^{2}$ en fase de finalización

\begin{tabular}{lcccccc}
\hline & $\begin{array}{c}\text { HT } \\
(\%)\end{array}$ & $\begin{array}{c}\text { HG } \\
(\mathrm{g} / \mathrm{dl})\end{array}$ & $\begin{array}{c}\text { GR } \\
\left(10^{3} / \mu \mathrm{l}\right)\end{array}$ & $\begin{array}{c}\text { Heterófilos } \\
(\%)\end{array}$ & $\begin{array}{c}\text { Linfocitos } \\
(\%)\end{array}$ & RHL \\
\hline Tratamientos & & & & & & \\
Sexo x Pienso x Alfalfa & & & & & & \\
Macho x 12 h/d x 0\% & $38.31^{\mathrm{a}}$ & 15.71 & 3314 & 58.60 & $22.82^{\mathrm{b}}$ & $2.57^{\mathrm{a}}$ \\
Macho x 12 h/d x 1\% & $35.52^{\mathrm{b}}$ & 15.64 & 3192 & 55.15 & $35.64^{\mathrm{a}}$ & $1.55^{\mathrm{b}}$ \\
Macho x 24 h/d x 0\% & $37.58^{\mathrm{a}}$ & 15.92 & 3308 & 50.31 & $38.17^{\mathrm{a}}$ & $1.32^{\mathrm{c}}$ \\
Macho x 24 h/d x 1\% & $35.19^{\mathrm{b}}$ & 14.81 & 3184 & 50.20 & $39.42^{\mathrm{a}}$ & $1.27^{\mathrm{c}}$ \\
Hembra x 12 h/d x 0\% & $34.98^{\mathrm{b}}$ & 15.07 & 3090 & 54.81 & $21.79^{\mathrm{b}}$ & $2.51^{\mathrm{a}}$ \\
Hembra x 12 h/d x 1\% & $34.87^{\mathrm{b}}$ & 15.09 & 3052 & 52.46 & $32.24^{\mathrm{a}}$ & $1.63^{\mathrm{b}}$ \\
& & & & & & \\
Hembra x 24 h/d x 0\% & $35.68^{\mathrm{b}}$ & 15.62 & 3118 & 51.72 & $34.31^{\mathrm{a}}$ & $1.51^{\mathrm{b}}$ \\
Hembra x 24 h/d x 1\% & $35.72^{\mathrm{b}}$ & 15.24 & 3191 & 48.98 & $36.72^{\mathrm{a}}$ & $1.33^{\mathrm{c}}$ \\
$\quad$ SEM & 1.266 & 0.137 & 33.54 & 1.134 & 2.389 & 0.186 \\
Efectos principales & & & & & & \\
Sexo & & & & & & \\
Macho & 36.65 & 15.52 & 3249 & 53.57 & $34.01^{\mathrm{a}}$ & 1.68 \\
Hembra & 35.31 & 15.26 & 3112 & 51.99 & $31.29^{\mathrm{b}}$ & 1.74 \\
Pienso & & & & & & \\
12 h/d & 35.92 & 15.38 & 3162 & 55.26 & $28.12^{\mathrm{b}}$ & $2.06^{\mathrm{a}}$ \\
24 h/d & 36.04 & 15.40 & 3200 & 50.30 & $37.18^{\mathrm{a}}$ & $1.36^{\mathrm{b}}$ \\
Alfalfa & & & & & & \\
0\% & 36.64 & 15.58 & 3207 & 53.86 & $29.30^{\mathrm{b}}$ & $1.98^{\mathrm{a}}$ \\
1\% & 35.33 & 15.20 & 3155 & 51.70 & $36.01^{\mathrm{a}}$ & $1.45^{\mathrm{b}}$ \\
p-value & & & & & & \\
Sexo x Pienso x Alfalfa & 0.043 & 0.749 & 0.274 & 0.069 & 0.043 & 0.037 \\
Sexo x Pienso & 0.273 & 0.659 & 0.381 & 0.172 & 0.096 & 0.132 \\
Sexo x Alfalfa & 0.301 & 0.573 & 0.275 & 0.283 & 0.136 & 0.095 \\
Pienso x Alfalfa & 0.174 & 0.398 & 0.258 & 0.159 & 0.075 & 0.085 \\
Sexo & 0.539 & 0.759 & 0.106 & 0.105 & 0.048 & 0.164 \\
Pienso & 0.653 & 0.641 & 0.163 & 0.063 & 0.017 & 0.017 \\
Alfalfa & 0.473 & 0.382 & 0.095 & 0.203 & 0.006 & 0.038 \\
\hline
\end{tabular}

${ }^{1}$ Cada valor representa la media de cuatro repeticiones para los efectos del tratamiento y 16 repeticiones para los efectos principales. Cada repetición estuvo conformada por 1 pavo

2 Los niveles de suplementación con alfalfa fresca fueron calculados en relación al peso corporal del pavo

SEM: Error estándar de la media. HT: Hematocrito. HG: Hemoglobina. GR: Glóbulos rojos $\mathrm{a}, \mathrm{b}, \mathrm{c}, \mathrm{d}$ Las medias dentro de una columna que no comparten igual superíndice difieren significativamente $(p<0.05)$ para los efectos del tratamiento y para los efectos principales 
hígado, y puede contrarrestar el estado de estrés del pavo ocasionado por la restricción del pienso.

- La restricción de horas de acceso al pienso y la suplementación con alfalfa del pavo en fase de crianza final no afectan el rendimiento de carcasa ni la acumulación de grasa abdominal en la canal.

\section{Literatura Citada}

1. Al-Murrani WK, Al-Rawi IK, Raof NM. 2002. Genetic resistance to Salmonella Typhimurium in two lines of chickens selected as resistant and sensitive on the basis of the heterophil/lymphocyte ratio. Brit Poultry Sci 43: 501-507. doi: 10.1080/000716602200-0004408

2. Balog JM, Kidd BD, Huff WE, Huff GR, Rath NC, Anthony NB. 2003. Effect of cold stress on broilers selected for resistance or susceptibility to ascites syndrome. Poultry Sci 82: 1383-1387. doi: $10.1093 / \mathrm{ps} / 82.9 .1383$

3. Bartz BM, Anderson KA, OviedoRondon EO, Livingtson K, Grimes JL. 2020. Effects of stocking density on large white, commercial tom turkeys reared to 20 weeks of age: 1 . Growth and performance. Poultry Sci 99: 55825586. doi: 10.1016/j.psj.2020.08.024

4. Beker A, Vanhooser SL, Swartzlander JH, Teeter RG. 2003. Graded atmospheric oxygen level effects on performance and ascites incidence in broilers. Poultry Sci 82: 1550-1553. doi: 10.1093/ $\mathrm{ps} / 82.10 .1550$

5. Chen CY, Lin HY, Chen YW, Ko YJ, Liu YJ, Chen YH, Walzem RL, Chen SE. 2017. Obesity-associated cardiac pathogenesis in broiler breeder hens: pathological adaption of cardiac hypertrophy. Poultry Sci 96: 2428-2437 doi: 10.3382/ps/pex015

6. Dunkley CS, McReynolds JL, Dunkley KD, Kubena LF, Nisbet DJ, Ricke SC. 2007. Molting in Salmonella enteritidis-challenged laying hens fed alfalfa crumbles. III. Blood plasma metabolite response. Poultry Sci 86: 2492-2501. doi: 10.3382/ps.2006-00400

7. Farghly MFA, Mahrose KM, Galal AE, Ali RM, Ahmad EAM, Rehman $Z U$, Ullah $Z$, et al. 2018. Implementation of different feed withdrawal times and water temperatures in managing turkeys during heat stress. Poultry Sci 97:3076-3084. doi: 10.3382/ps/pey173

8. Fatkin D, Graham RM. 2002. Molecular mechanisms of inherited cardiomyo-pathies. Physiol Rev 82: 945980. doi: 10.1152/physrev.00012.2002

9. Ferket PR, Malheiros RD, Moraes VMB, Ayoola AA, Barasch I, Toomer OT, Torrent J. 2020. Effects of functional oils on the growth, carcass and meat characteristics, and intestinal morphology of commercial turkey toms. Poultry Sci 99: 3752-3760. doi: 10.1016/ j.psj.2020.03.050

10. Ferrante V, Lolli S, Ferrari L, Watanabe TTN, Tremolada C, Marchewka J, Estevez I. 2019. Differences in prevalence of welfare indicators in male and female turkey flocks (Meleagris gallopavo). Poultry Sci 98: 1568-1574. doi: $10.3382 / \mathrm{ps} /$ pey534

11. Frame DD, Hooge DM, Cutler R. 2001. Interactive effects of dietary sodium and chloride on the incidence of spontaneous cardiomyopathy (round heart) in turkeys. Poultry Sci 80: 15721577. doi: $10.1093 / \mathrm{ps} / 80.11 .1572$

12. Genao A, Seth K, Schmidt U, Carles M, Gwathmey JK. 1996. Dilated cardiomyopathy in turkeys: an animal model for the study of human heart failure. Lab Anim Sci 46: 399-404.

13. González-Quintanilla Y, Cuevas E, Cruz-Lumbreras R, Carrillo-Castilla P, Rodríguez-Antolín J, MartínezGómez M. 2014. Relación entre células sanguíneas y variables metabólicas en mujeres indígenas de diferentes edades que viven a gran altitud. Rev Espec Cienc Quím-Biol 17: 111-116. 
14. Hao X, Zhang S, Cheng X, Li M, Sun T, Zhang J, Guo W, Li L. 2013. Imidapril inhibits right ventricular remodeling induced by low ambient temperature in broiler chickens. Poultry Sci 92: 14921497. doi: 10.3382/ps.2012-02671

15. Huff GR, Huff WE, Balog JM, Rath NC, Anthony NB, Nestor KE. 2005. Stress response differences and disease susceptibility reflected by heterophil to lymphocyte ratio in turkeys selected for increased body weight. Poultry Sci 84 : 709-717. doi: $10.1093 / \mathrm{ps} / 84.5 .709$

16. Huffman K, Zapata I, Reddish JM, Lilburn MS, Wick M. 2012. Feed restriction delays developmental fast skeletal muscle myosin heavy chain isoforms in turkey poults selected for differential growth. Poultry Sci 91:31783183. doi: 10.3382/ps.2012-02367

17. Jankowski J, Mikulski D, Mikulska M, Ognik K, Calyniuk Z, Mroz E, Zdunczyk Z. 2020. The effect of different dietary ratios of arginine, methionine, and lysine on the performance, carcass traits, and immune status of turkeys. Poultry Sci 99: 1028-103. doi: 10.1016/j.psj.2019.10.008

18. Kamely M, Torshizi MAK, Rahimi S. 2015. Incidence of ascites syndrome and related hematological response in shortterm feed-restricted broilers raised at low ambient temperature. Poultry Sci 94: 2247-2256. doi: 10.3382/ps/pev197

19. Kwiecieñ M, Winiarska-Mieczan A, Danek-Majewska A, Kwiatkowska K, Krusiñski R. 2021. Effects of dietary alfalfa protein concentrate on lipid metabolism and antioxidative status serum, and composition and fatty acids profile, antioxidative status and dietetic value of the muscles in broiler. Poultry Sci 100: 100974. doi: 10.1016/ j.psj.2020.12.071

20. Laudadio V, Tufarelli V, Dario $M$, D'Emilio FP, Vicenti A. 2009. Growth performance and carcass characteristics of female turkeys as affected by feeding programs. Poultry Sci $88: 805-810$. doi: 10.3382/ps.2008-00082
21. Lin KC, Gyenai K, Pyle RL, Geng T, Xu J, Smith EJ. 2006. Candidate gene expression analysis of toxin-induced dilated cardiomyopathy in the turkey (Meleagris gallopavo). Poultry Sci 85:2216-2221.

22. Luger D, Shinder D, Wolfenson D, Yahav S. 2003. Erytropoiesis regulation during the development of ascites syndrome in broiler chickens - A possible role of corticosterone. J Anim Sci 81: 784790. doi: $10.2527 / 2003.813784 \mathrm{x}$

23. Marchewka J, Watanabe TTN, Ferrante V, Estevez L. 2019. Welfare assessment in broiler farms: Transect walks versus individual scoring. Poultry Sci 92: 2588-2599. doi: 10.3382/ps.201303229

24. Marrett LE, Robb EJ, Frank RK. 2000. Efficacy of neomycin sulfate water medication on the control of mortality associated with colibacillosis in growing turkeys. Poultry Sci 79: 12-17. doi: $10.1093 / \mathrm{ps} / 79.1 .12$

25. [MINAGRI] Ministerio de Desarrollo Agrario y Riego. 2020. Producción y comercialización de productos avícolas. Lima: Boletín Estadístico Mensual - Junio. 21 pp. Disponible en: https:// cdn.www.gob.pe/uploads/document/file/ 1249491/producci\%C3\%B3n-comercializaci\%C3\%B3n-avicola-jun-2020190820.pdf

26. Özkan S, Takma C, Yahav S, Sögüt B, Türkmut L, Erturun H, Cahaner A. 2010. The effects of feed restriction and ambient temperature on growth and ascites mortality of broilers reared at high altitude. Poultry Sci 89: 974-985. doi: 10.3382/ps.2009-00293.

27. Peñaloza D, Arias S. 2011. Corazón y circulación pulmonar en grandes alturas: nativos normales y mal de montaña crónico. Rev Per Cardiol 37: 38-56.

28. Petracci M, Bianchi M, Cavani C, Gaspari P, Lavazza A. 2006. Preslaughter mortality in broiler chickens, turkeys, and spent hens under commercial slaughtering. Poultry Sci 85 : $1660-$ 1664. doi: $10.1093 / \mathrm{ps} / 85.9 .1660$ 
29. Poller W, Kuhl U, Tschoepe $C$, Pauschinger M. 2005. Genomeenvironment interactions in the molecular pathogenesis of dilated cardiomyopathy. J Mol Med 83: 579-586. doi: 10.1007/ s00109-005-0664-2

30. Rathgeber BM, MacIsaac JL, MacKenzie ME. 2007. Feeding turkeys a highly digestible supplement during preslaughter feed withdrawal. Poultry Sci 86: 2029-2033. doi: 10.1093/ps/ 86.9.2029

31. Roy RD, Edens FW, Parkhurst CR, Qureshi MA, Havenstein GB. 2002. Influence of a propionic acid feed additive on performance of turkey poults with experimentally induced poult enteritis and mortality syndrome. Poultry Sci 81:951-957.

32. Scanes CG, Hurst K, Thaxton $Y$, Archer GS, Johnson A. 2020. Effect of transportation and shackling on plasma concentrations of corticosterone and heterophil to lymphocyte ratios in market weight male turkeys in a commercial operation. Poultry Sci 99:546-554. doi: $10.3382 / \mathrm{ps} / \mathrm{pez} 485$

33. Singh AK, Tiwari UP, Berrocoso JD, Dersjant-Li Y, Awati A, Jha R. 2019. Effects of a combination of xylanase, amylase and protease, and probiotics on major nutrients including amino acids and non-starch polysaccharides utilization in broilers fed different level of fibers. Poultry Sci 98: 5571-5581. doi: 10.1093/ ps/81.7.951

34. Terry VK, Figueroa E, Fuentes $N E$, Ayón M, Guzmán J, Carcelén F. 2012. Peso corporal, índices cardíacos, hematocrito y hemoglobina en dos líneas comerciales de pavos. Rev Inv Vet Perú 23:510-515.

35. Vasdal G, Marchewka J, Moe RO. 2021. Associations between animalbased measures at $11 \mathrm{wk}$ and slaughter data at $20 \mathrm{wk}$ in turkey toms (Meleagris gallopavo). Poultry Sci 100: 412-419. doi: 10.1016/j.psj.2020.11.010.

36. Vecerek $\boldsymbol{V}$, Vecerkova L, Voslarova $\boldsymbol{E}$. 2019. Comparison of the frequency of patho-anatomic findings in laying hens with findings in broiler chickens and turkeys detected during post-mortem veterinary inspection. Poultry Sci 98: 5385-5391. doi: 10.3382/ps/pez364

37. Velleman S, Clark DL, Tonniges JR. 2019. The effect of nutrient restriction on the proliferation and differentiation of turkey pectoralis major satellite cells differing in age and growth rate. Poultry Sci 98: 1893-1902. doi: 10.3382/ps/ pey509

38. Vizcarra FR, Verghese M, Vizcarra JA. 2018. Effect of short- and long-term feed restriction on ghrelin concentrations in turkeys. Poultry Sci 97: 2183-2188. doi: $10.3382 / \mathrm{ps} / \mathrm{pey} 039$

39. Wideman RF. 2000. Cardio-pulmonary hemodynamics and ascites in broiler chickens. Avian Poult Biol Rev 11: 24-43.

40. Yahav S, Straschnow A, Plavnik I, Hurwitz S. 1997. Blood system response of chickens to changes in environmental temperature. Poultry Sci 76: 627633. doi: $10.1093 / \mathrm{ps} / 76.4 .627$

41. Zepeda EA, Kooyman DL. 2002. Breeding trial based on RAPD analysis of turkey spontaneous cardiomyopathy. In: Plant, Animal, Microbe Genomes X Conference. San Diego, CA. 